\title{
A New Approach to Risk Comparison via Uncertain Measure
}

\author{
Shengguo Li \\ School of Mathematics and Statistics, Huazhong Normal University, Hubei, China \\ Jin Peng* \\ Institute of Uncertain Systems, Huanggang Normal University, Hubei, China
}

(Received: February 10, 2012 / Revised: April 19, 2012 / Accepted: April 23, 2012)

\begin{abstract}
This paper presents a new approach to risk comparison in uncertain environment. Based on the uncertainty theory, some uncertain risk measures and risk comparison rules are proposed. Afterward the bridges are built between uncertain risk measures and risk comparison rules. Finally, several comparable examples are given.
\end{abstract}

Keywords: Uncertainty Theory, Uncertain Measure, Uncertainty Distribution, Risk Analysis

* Corresponding Author, E-mail: pengjin01@tsinghua.org.cn

\section{INTRODUCTION}

As we all know, risk is an important factor in making decisions. In many areas, decision makers must often choose a course of action in the face of risks. In order to monitor risks timely and minimize the loss caused by risk, it is necessary to analyze and compare a great variety of risks.

There exist many ways to depict and measure risk because every decision maker has its own perception of risk. Variance and standard deviation were the traditional risk measures and first applied in the selection of portfolios by Markowitz (1952). After about 40 years, value at risk (VaR) was introduced in financial risk management by Guldimann (1995). Now, it is adopted by many financial institutions to control the risk of loss. Unfortunately, value at risk does not possess subadditivity. Therefore, tail value at risk (TVaR) as a natural remedy for the shortcoming of $\mathrm{VaR}$ was proposed by Artzner et al. (1997). These risk measures have become important tools in the stochastic risk analysis fields. In the stochastic environment, risk usually was regarded as a random variable and there were many methods to rank the random variables. The applications of stochastic dominance can be found in welfare, poverty and inequality, such as
Shaked and Shanthikumar (1994), Barrett and Donald (2003).

In fuzzy environment, many researchers regarded risk as a fuzzy variable and adopted fuzzy theory and methodology as an approach to risk analysis (Zmeskal, 2005; Lee and Chen, 2008). From the beginning of the development of fuzzy set theory (Zadeh, 1965), the problem of fuzzy variable dominance was studied. Lee and Li (1988) proposed comparison of fuzzy numbers based on the probability measure of fuzzy events. Tran and Duckstein (2002) compared fuzzy numbers using a fuzzy distance measure. Nojavan and Ghazanfari (2006) presented fuzzy ranking method by desirability index. Peng et al. (2005) and Peng et al. (2007) provided a fuzzy dominance method based on credibility measure. Recently, Peng (2008) presented the concept of credibilistic VaR via credibility theory to measure fuzzy risk. Afterward, Peng (2009b) proposed average value at risk (AVaR) in fuzzy risk analysis. These methods have played important roles in fuzzy risk analysis problems.

However, many surveys showed that some imprecise phenomena behave neither like randomness nor like fuzziness. In order to deal with this uncertainty different from randomness and fuzziness, Liu (2007) founded uncertainty theory based on normality, self-duality, coun- 
table subadditivity, and product measure axioms in 2007, and it was refined by Liu (2010a). As a new tool to study the uncertainty in human systems, uncertainty theory motivates a new area of risk analysis called uncertain risk analysis. Here the risk is defined as the accidental loss plus uncertain measure of such loss (Liu, 2010b). The fact that risk is usually not a known constant shows that it is reasonable to represent risk by uncertain variable. Up to now, many significant risk measures have been proposed from different angles in uncertain risk analysis. Peng (2009a) suggested the VaR and TVaR based on uncertainty theory. Liu (2010b) provided the risk index to measure some risk loss. Peng and Li (2010, 2011) studied the distortion risk measure and spectral measure of uncertain risk, respectively. These uncertain measures can be widely used as tools of risk analysis in uncertain environment. In this paper, we aim to define some comparison rules of risks within the framework of uncertainty theory and discuss the relationship between these comparison rules and uncertain risk measures. The risk comparison approach is different from those in stochastic and fuzzy environment. It is worthwhile to compare risks on the basis of these uncertain measures.

The remainder of this paper is organized as follows. Section 2 presents preliminaries in the uncertainty theory. The concepts of some risk measures based on the uncertainty theory are given in section 3 . Section 4 introduces three types of comparison rules of risks. In section 5, we build the bridges between uncertain risk measures and comparison rules of risks. Section 6 gives some comparable illustrations. The last section contains some concluding remarks.

\section{PRELIMINARIES}

Let $\Gamma$ be a nonempty set. A collection $L$ of subsets of $\Gamma$ is a $\sigma$-algebra. Uncertain measure $M$ introduced by Liu (2007) is a set function if it satisfies the following axioms:

(1) (Normality) $M\{\Gamma\}=1$;

(2) (Self-duality) $M\{\Lambda\}+M\left\{\Lambda^{c}\right\}=1$ for any $\Lambda \in L$;

(3) (Subadditivity) For every countable sequence of events $\left\{\Lambda_{i}\right\}$, we have

$$
M\left\{\cup \Lambda_{i}\right\} \leq \sum_{i} M\left\{\Lambda_{i}\right\}
$$

The triplet $(\Gamma, L, M)$ is called an uncertain space. In order to obtain an uncertain measure of compound event, Liu (2009) defined a product uncertain measure which produces the forth axiom of uncertainty theory:

(4) (Product axiom) Let $\left(\Gamma_{k}, L_{k}, M_{k}\right)$ be uncertain space for $k=1,2, \cdots, n$. Then the product uncertain measure $M$ is an uncertain measure on the product $\sigma$ algebra $\prod L_{k}$ satisfying

$$
M\left\{\prod_{k=1}^{n} \Lambda_{k}\right\}=\min _{1 \leq k \leq n} M\left\{\Lambda_{k}\right\} .
$$

An uncertain variable is defined as a measurable function from an uncertain space $(\Gamma, L, M)$ to the set of real numbers. The uncertainty distribution $\Phi: \mathfrak{R} \rightarrow[0,1]$ of an uncertain variable $\xi$ is defined by Liu (2007) as

$$
\Phi(x)=M\{\gamma \in \Gamma \mid \xi(\gamma) \leq x\}
$$

and the inverse function $\Phi^{-1}$ is called the inverse uncertainty distribution of $\xi$.

Expected value is the average value of uncertain variable in the sense of uncertain measure, and represents the size of uncertain variable. The expected value of uncertain variable $\xi$ is defined by Liu (2007) as

$$
E[\xi]=\int_{0}^{+\infty} M\{\xi \geq \gamma\} d \gamma-\int_{-\infty}^{0} M\{\xi \leq \gamma\} d \gamma
$$

provided that at least one of the two integrals is finite.

Liu (2010a) has proved that

$$
E[\xi]=\int_{0}^{1} \Phi^{-1}(\alpha) d \alpha
$$

if the expected value of uncertain variable $\xi$ exists.

Let $\xi$ be an uncertain variable with finite expected value $E[\xi]$. The variance of $\xi$ is defined as $V[\xi]=E$ $\left[(\xi-E[\xi])^{2}\right]$. And the standard deviation of $\xi$ is defined as $\sqrt{V[\xi]}$.

An uncertain variable $\xi$ is called linear if it has a linear uncertainty distribution

$$
\Phi(x)=\left\{\begin{array}{cc}
0, & x<a \\
\frac{x-a}{b-a}, & a \leq x<b \\
1, & x \geq b,
\end{array}\right.
$$

denoted by $L(a, b)$ where $a$ and $b$ are real numbers with $a<b$, and the inverse uncertainty distribution is

$$
\Phi^{-1}(\alpha)=(1-\alpha) a+\alpha b, \quad 0<\alpha<1 .
$$

The expected value and variance are $(a+b) / 2$ and $(b-a)^{2} / 12$.

An uncertain variable $\xi$ is called normal if it has a normal uncertainty distribution

$$
\Phi(x)=\left(1+\exp \left(\frac{\pi(e-x)}{\sqrt{3} \sigma}\right)\right)^{-1}, \quad x \in \mathfrak{R},
$$

denoted by $N(e, \sigma)$, where $e$ and $\sigma$ are real numbers with $\sigma>0$, and the inverse uncertainty distribution is

$$
\Phi^{-1}(\alpha)=e+\frac{\sqrt{3} \sigma}{\pi} \ln \frac{\alpha}{1-\alpha}, \quad 0<\alpha<1 .
$$

The expected value and variance are $e$ and $\sigma^{2}$. 


\section{SOME RISK MEASURES IN UNCERTAINTY ENVIRONMENT}

Risk measure is the core of risk analysis. Here we introduce some uncertain risk measures quoted from Peng (2009a, 2009b).

Definition 1: (Peng, 2009a) Let $\xi$ be an uncertain variable and $\alpha \in(0,1)$ be the risk confidence level. Then the function $\xi_{\mathrm{VaR}}:(0,1) \rightarrow \mathfrak{R}$ such that

$$
\xi_{\text {VaR }}(\alpha)=\inf \{x \mid M\{\xi \leq x\} \geq \alpha\} .
$$

From this definition, we can see that $\xi_{\mathrm{VaR}}$ just is the generalized inverse function of $\Phi^{-1}(\alpha)$.

The VaR measures of linear uncertain variable $\xi$ and normal uncertain variable $\eta$ are

$$
\xi_{\text {VaR }}(\alpha)=(1-\alpha) a+\alpha b
$$

and

$$
\eta_{\text {VaR }}(\alpha)=e+\frac{\sqrt{3} \sigma}{\pi} \ln \frac{\alpha}{1-\alpha}
$$

Definition 2: (Peng, 2009b) Let $\xi$ be an uncertain variable and $\alpha \in(0,1)$ be the risk confidence level. Then the function $\xi_{\mathrm{AVaR}}:(0,1) \rightarrow \mathfrak{R}$ such that

$$
\xi_{\text {AVaR }}(\alpha)=\frac{1}{\alpha} \int_{0}^{\alpha} \xi_{\text {VaR }}(\beta) d \beta .
$$

The AVaR measures of linear uncertain variable $\xi$ and normal uncertain variable $\eta$ are

$$
\xi_{\text {AVaR }}(\alpha)=a+\frac{1}{2}(b-a) \alpha,
$$

and

$$
\eta_{\text {AVaR }}(\alpha)=e+\frac{\sqrt{3} \sigma}{\pi}\left[\ln \alpha+\frac{1-\alpha}{\alpha} \ln (1-\alpha)\right]
$$

Definition 3: (Peng, 2009a) Let $\xi$ be an uncertain variable and $\alpha \in(0,1)$ be the risk confidence level. Then the function $\xi_{\mathrm{TVaR}}:(0,1) \rightarrow \mathfrak{R}$ such that

$$
\xi_{\text {TVaR }}(\alpha)=\frac{1}{1-\alpha} \int_{\alpha}^{1} \xi_{\text {VaR }}(\beta) d \beta .
$$

The TVaR measures of linear uncertain variable $\xi$ and normal uncertain variable $\eta$ are

$$
\xi_{\text {TVaR }}(\alpha)=a+\frac{1}{2}(b-a)(1+\alpha)
$$

and

$$
\eta_{\text {TVaR }}(\alpha)=e-\frac{\sqrt{3} \sigma}{\pi}\left[\ln (1-\alpha)+\frac{\alpha}{1-\alpha} \ln (\alpha)\right] .
$$

A disadvantage of $\mathrm{VaR}$ is that it does not give the information about the severity of loss within and beyond the VaR level. Then, AVaR and TVaR mend this shortcoming. AVaR gives the estimation of loss within the VaR level and TVaR accounts for the severity of loss failure and not only the chance of failure. AVaR and $\mathrm{TVaR}$ are considered to provide the better measures of risk. Moreover, it can be verified that they all possess the properties of monotonicity, positive homogeneity, translation invariance, independence additivity.

\section{COMPARISON OF UNCERTAIN RISKS}

Let $\xi$ and $\eta$ be two risks treated as uncertain variables with distribution functions $\Phi(x)$ and $\Psi(x)$, respectively. This section will describe three types of rules to compare risks.

Definition 4: (First comparison rule) Let $\xi$ and $\eta$ be two risks with distribution functions $\Phi(x)$ and $\Psi(x)$, respectively. $\xi$ is said to be smaller than $\eta$ in first-order, if and only if $\Phi(x) \leq \Psi(x)$ for all $x \in \mathfrak{R}$, written $\xi \preceq_{R C 1} \eta$.

First comparison rule shows the possibility of same loss is small if the distribution function of risk always lies to the lower-right of the other. We can verify that the first comparison rule is reflexive, transitive and antisymmetric. That is, this rule is a partial order. Consequently, it is essential to introduce more applicable rules.

Definition 5: (Second comparison rule) Let $\xi$ and $\eta$ be two risks with distribution functions $\Phi(x)$ and $\Psi(x)$, respectively. $\xi$ is said to be smaller than $\eta$ in secondorder, if and only if $\int_{-\infty}^{x} \Phi(t) d t \leq \int_{-\infty}^{x} \Psi(t) d t$ for all $x \in \mathfrak{R}$, written $\xi \preceq_{R C 2} \eta$.

Definition 6: (Third comparison rule) Let $\xi$ and $\eta$ be two risks with distribution functions $\Phi(x)$ and $\Psi(x)$, respectively. $\xi$ is said to be smaller than $\eta$ in thirdorder, if and only if $\int_{x}^{+\infty}(1-\Phi(t)) d t \leq \int_{x}^{+\infty}(1-\Psi(t)) d t$ for all $x \in \mathfrak{R}$, written $\xi \preceq_{R C 3} \eta$.

It is clear that the second and third comparison rules also meet reflexivity and transitivity. In addition, $\xi \preceq_{R C 1} \eta$ implies both $\xi \preceq_{R C 2} \eta$ and $\xi \preceq_{R C 3} \eta$.

\section{BRIDGES BETWEEN UNCERTAIN MEASURES AND UNCERTAIN RISK COMPARISONS}

In this section, we will investigate the relationships between uncertain measures and risk comparison rules. 
Theorem 1: Let $\xi$ and $\eta$ be two risks with continuous distribution functions $\Phi(x)$ and $\Psi(x)$, respectively. Then $\xi \preceq_{R C 1} \eta$ iff $\xi_{\mathrm{VaR}}(\alpha) \geq \eta_{\text {VaR }}(\alpha)$ for all $\alpha \in(0,1)$.

\section{Proof:}

Necessity: If $\xi \preceq_{R C 1} \eta$, i.e., $\Phi(x) \leq \Psi(x)$ for all $x \in \mathfrak{R}$, we have inf $\{x \mid \Phi(x) \geq \alpha\} \geq \inf \{x \mid \Psi(x) \geq \alpha\}$ for all $\alpha$, which means $\xi_{\mathrm{VaR}}(\alpha) \geq \eta_{\text {VaR }}(\alpha)$.

Sufficiency: For any $x \in \mathfrak{R}$, there is $\alpha \in(0,1)$ such that $\xi_{\mathrm{VaR}}(\alpha)=x . \xi_{\mathrm{VaR}}(\alpha) \geq \eta_{\text {VaR }}(\alpha)$ shows $\Phi(x)=\alpha \leq \Psi(x)$.

Theorem 2: Let $\xi$ and $\eta$ be two risks with continuous distribution functions $\Phi(x)$ and $\Psi(x)$, respectively. Then $\xi \preceq_{R C 2} \eta$ iff $\xi_{\mathrm{AVaR}}(\alpha) \geq \eta_{\text {AVaR }}(\alpha)$ for all $\alpha \in(0,1)$.

\section{Proof:}

Necessity: Assuming that $\xi \preceq_{R C 2} \eta$ and $\alpha \in(0,1)$, we construct the function

$$
f(a)=\int_{-\infty}^{a} \Phi(t) d t-\alpha a, a \in \mathfrak{R} .
$$

Next, we will prove that $f(a)$ takes the minimum value at $a=\Phi^{-1}(\alpha)=\xi_{\text {VaR }}(\alpha)$. On one hand, the inequality

$$
\int_{\Phi^{-1}(\alpha)}^{a} \Phi(t) d t \geq \int_{\Phi^{-1}(\alpha)}^{a} \alpha d t
$$

always holds for $a \geq \Phi^{-1}(\alpha)$. Then,

$$
\int_{-\infty}^{a} \Phi(t) d t-\alpha a \geq \int_{-\infty}^{\Phi^{-1}(\alpha)} \Phi(t) d t-\alpha \Phi^{-1}(\alpha)
$$

i.e.,

$$
f(a) \geq f\left(\Phi^{-1}(\alpha)\right) .
$$

On the other hand, for $a \leq \Phi^{-1}(\alpha)$, we have

$$
\int_{a}^{\Phi^{-1}(\alpha)} \Phi(t) d t \leq \int_{a}^{\Phi^{-1}(\alpha)} \alpha d t
$$

i.e.,

$$
f(a) \geq f\left(\Phi^{-1}(\alpha)\right) .
$$

That is to say, $f(a) \geq f\left(\Phi^{-1}(\alpha)\right)$ for any $a \in \mathfrak{R}$. Then we have

$$
\begin{aligned}
\xi_{\text {AVaR }}(\alpha) & =\xi_{\text {VaR }}-\frac{1}{\alpha} \int_{-\infty}^{\xi_{\text {VaR }}(\alpha)} \Phi(t) d t \\
& =-\frac{f\left(\Phi^{-1}(\alpha)\right)}{\alpha} \\
& \geq-\frac{f\left(\Psi^{-1}(\alpha)\right)}{\alpha} t \\
& =\eta_{\text {VaR }}(\alpha)-\frac{1}{\alpha} \int_{-\infty}^{\Psi^{-1}(\alpha)} \Phi(t) d t
\end{aligned}
$$

$$
\begin{aligned}
& \geq \eta_{\text {VaR }}(\alpha)-\frac{1}{\alpha} \int_{-\infty}^{\Psi^{-1}(\alpha)} \Psi(t) d t \\
& =\eta_{\text {AVaR }}(\alpha) .
\end{aligned}
$$

Sufficiency: Assume that $\xi_{\mathrm{AVaR}}(\alpha) \geq \eta_{\text {AVaR }}(\alpha)$ for all $\alpha \in$ $(0,1)$. For a such that $0<\Phi(a)<1$, we have

$$
\begin{aligned}
\int_{-\infty}^{a} \Phi(t) d t & =a \Phi(a)-\int_{0}^{\Phi^{-1}(a)} \Phi^{-1}(\beta) d \beta \\
& =\Phi(a)\left(a-\xi_{\text {AVaR }}(\Phi(a))\right) \\
& \leq \Phi(a)\left(a-\eta_{\text {AVaR }}(\Phi(a))\right) \\
& =\int_{-\infty}^{a} \Psi(t) d t+\int_{\Phi(a)}^{\Psi(a)}\left(\Psi^{-1}(\beta)-a\right) d \beta .
\end{aligned}
$$

By the $\alpha \leq \Psi(a) \Leftrightarrow a \geq \Psi^{-1}(\alpha)$, it is obvious that

$$
\int_{\Phi(a)}^{\Psi(a)}\left(\Psi^{-1}(\beta)-a\right) d \beta \leq 0
$$

which implies $\int_{-\infty}^{x} \Phi(t) d t \leq \int_{-\infty}^{x} \Psi(t) d t$ for a such that $0<$ $\Phi(a)<1$. It is easy to verify this inequality holds when $\Phi(a)=0$ and $\Phi(a)=1$. Hence, $\xi_{R C 2} \eta$ is proved.

Theorem 3: Let $\xi$ and $\eta$ be two risks with continuous distribution functions $\Phi(x)$ and $\Psi(x)$, respectively. Then $\xi \preceq_{R C 3} \eta$ iff $\xi_{\text {TVaR }}(\alpha) \leq \eta_{\text {TVaR }}(\alpha)$ for all $\alpha \in(0,1)$.

\section{Proof:}

Similarly, the necessity can be proved by constructing the function

$$
f(a)=(1-\alpha) a-\int_{a}^{+\infty} \Phi(t) d t, a \in \Re .
$$

And, we may prove the sufficiency after noticing

$$
\int_{a}^{+\infty} \Phi(t) d t=(1-\Phi(a))\left(\xi_{\text {TVaR }}(\Phi(a))-1\right) .
$$

\section{EXAMPLES}

In the uncertainty theory, many types of uncertain variables can be used to describe risks. In this section, we concentrate on the comparison of risks with linear and normal uncertain distributions. First, according to the three risk comparison rules, we can obtain the following results for two risks with linear uncertain distributions.

Theorem 4: Let $\xi \sim L\left(a_{1}, b_{1}\right)$ and $\eta \sim L\left(a_{2}, b_{2}\right)$ be two risks with $b_{1}-a_{1}<b_{2}-a_{2}$. Then

(1) $\xi \preceq_{R C 1} \eta$ iff $a_{1} \geq a_{2}, b_{1} \geq b_{2}$,

(2) $\xi \preceq_{R C 2} \eta$ iff $E[\xi] \geq E[\eta]$,

(3) $\xi \preceq_{R C 3} \eta$ iff $E[\xi] \leq E[\eta]$. 
Proof:

(1) It directly follows from the uncertain distribution of linear uncertain variable and definition of the first comparison rule. The result can be described in Figure 1 .

$\Phi(x)$

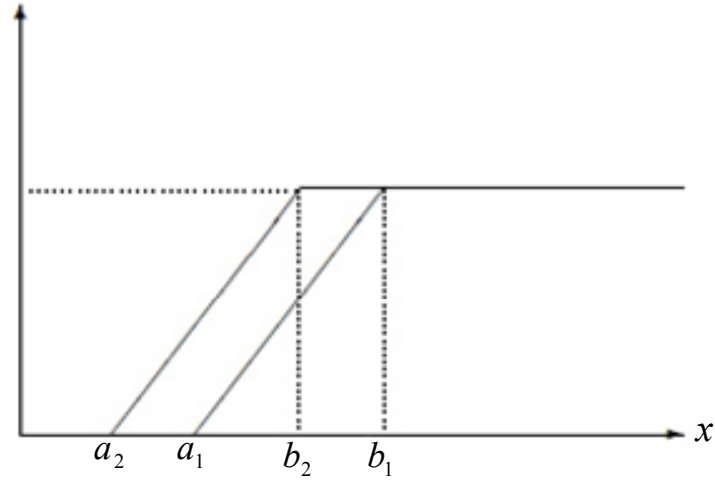

Figure 1. First comparison of linear risks.

(2) It follows from the second comparison rule and Theorem 2 that

$$
a_{1}+\frac{1}{2}\left(b_{1}-a_{1}\right) \alpha \geq a_{2}+\frac{1}{2}\left(b_{2}-a_{2}\right) \alpha \text {. }
$$

Under the hypothesis $b_{1}-a_{1}<b_{2}-a_{2}$, we get

$$
\alpha \leq \frac{2\left(a_{2}-a_{1}\right)}{\left(b_{1}-a_{1}\right)-\left(b_{2}-a_{2}\right)}
$$

which yields $b_{1}+a_{1} \geq b_{2}+a_{2}$, i.e., $E[\xi] \geq E[\eta]$. Then the result can be described in Figure 2 when $b_{2}<b_{1}$ and Figure 3 when $b_{1}<b_{2}$.

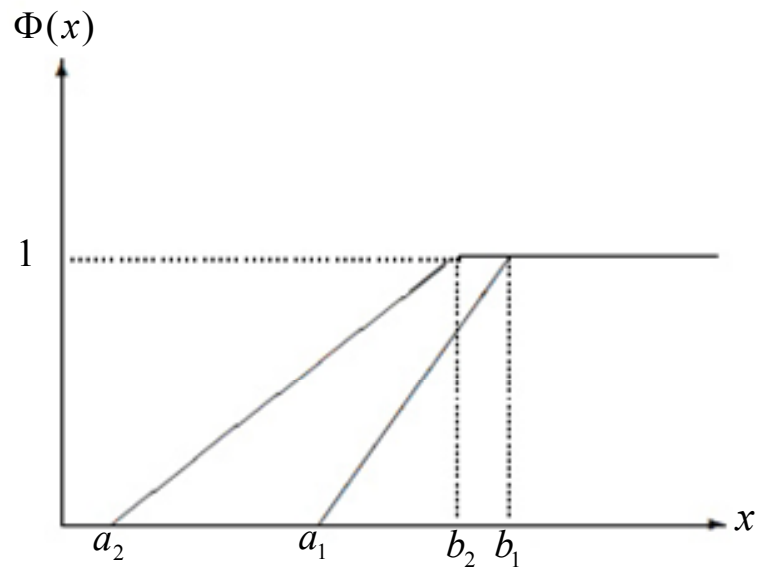

Figure 2. Second comparison of linear risks; $a_{2}<a_{1}$, $b_{1}>b_{2}$.

(3) By the analysis similar to (2), we have that $\xi \preceq_{R C 3} \eta$ if and only if $E[\xi] \leq E[\eta]$. We can describe the re- sult in Figure 4 when $a_{1}<a_{2}$ and Figure 5 when $a_{2}$ $<a_{1}$.

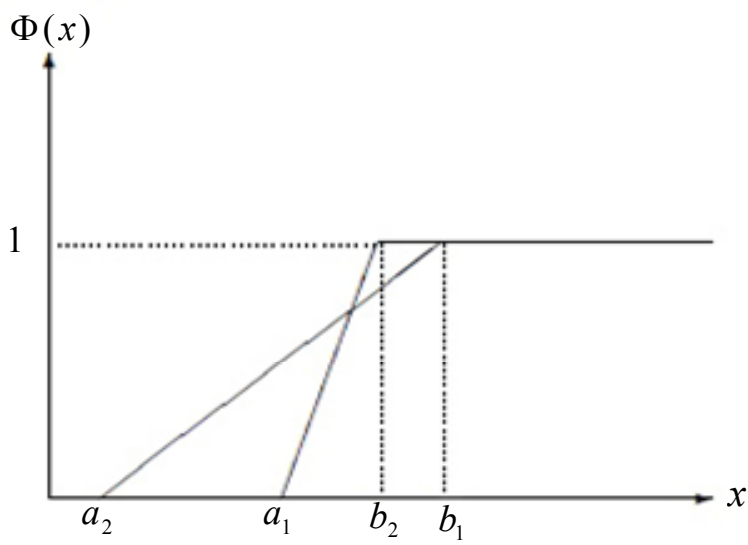

Figure 3. Second comparison of linear risks; $a_{2}<a_{1}$, $b_{1}<b_{2}$.

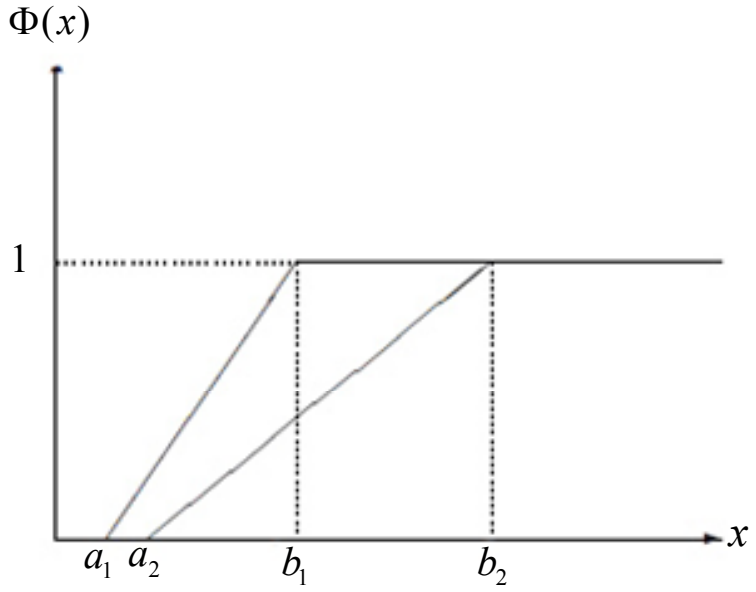

Figure 4. Third comparison of linear risks; $a_{1}<a_{2}, b_{1}<b_{2}$.

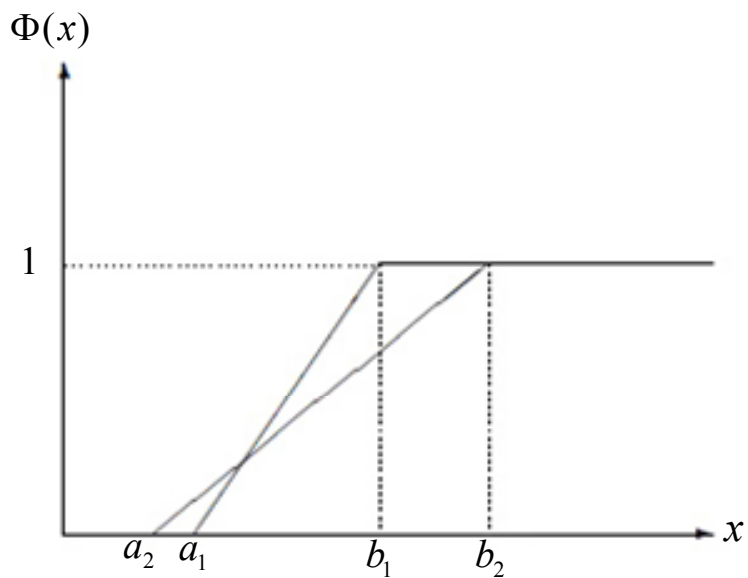

Figure 5. Third comparison of linear risks; $a_{2}<a_{1}$, $b_{1}<b_{2}$.

Next, we give the comparison results for two risks 
with normal uncertain distributions.

Theorem 5: Let $\xi \sim N\left(e_{1}, \sigma_{1}\right)$ and $\eta \sim N\left(e_{2}, \sigma_{2}\right)$. Then

(1) $\xi \preceq_{R C 1} \eta$ iff $e_{1} \geq e_{2}, \sigma_{1}=\sigma_{2}$,

(2) $\xi \preceq_{R C 2} \eta$ iff $e_{1} \geq e_{2}, \sigma_{1} \leq \sigma_{2}$,

(3) $\xi \preceq_{R C 3} \eta$ iff $e_{1} \leq e_{2}, \sigma_{1} \leq \sigma_{2}$.

\section{Proof:}

(1) It follows from the first comparison rule that $\xi_{R C 1} \eta$ if and only if $\Phi(x) \leq \Psi(x)$ for all $x \in \mathfrak{R}$ i.e.,

$$
\frac{\pi\left(e_{1}-x\right)}{\sqrt{3} \sigma_{1}} \geq \frac{\pi\left(e_{2}-x\right)}{\sqrt{3} \sigma_{2}}
$$

for any $x \in \mathfrak{R}$ which shows $e_{1} \geq e_{2}$ and $\sigma_{1}=\sigma_{2}$.

We know that the variance of an uncertain variable provides measure of the spread of the distribution around its expected value. So $\sigma_{1}=\sigma_{2}$ implies the same spread of the distributions around theirs respective expected values. The result can be described in Figure 6 .

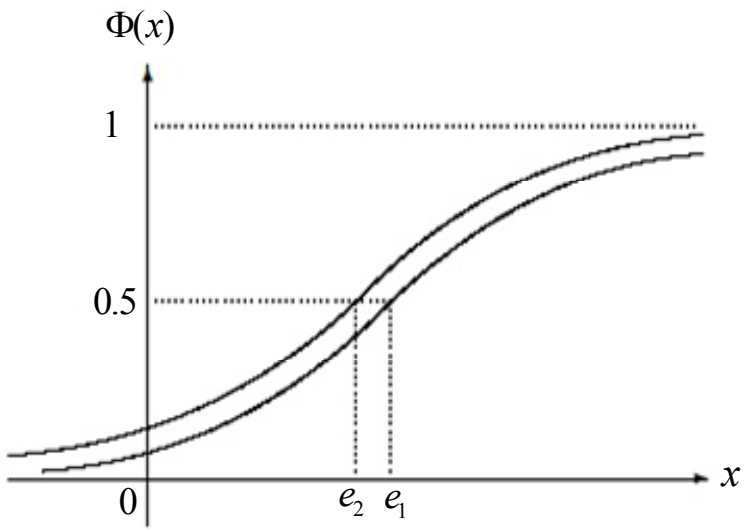

Figure 6. First comparison of normal risks.

(2) It follows from the second comparison rule and Theorem 2 that for $\alpha \in(0,1), f(\alpha)\left(\sigma_{2}-\sigma_{1}\right) \leq\left(e_{1}-e_{2}\right)$ where

$$
f(\alpha)=\frac{\sqrt{3}}{\pi}\left[\ln \alpha+\frac{1-\alpha}{\alpha} \ln (1-\alpha)\right] .
$$

So, we can obtain that $\xi \preceq_{R C 2} \eta$ iff $e_{1} \geq e_{2}$ and $\sigma_{1} \leq \sigma_{2}$.

In addition, $\sigma_{1} \leq \sigma_{2}$ indicates that the distribution of risk $\xi$ is more tightly concentrated around its expected value. The result can be described in Figure 7.

(3) It follows from the third comparison rule and Theorem 3 that for $\alpha \in(0,1), f(\alpha)\left(\sigma_{2}-\sigma_{1}\right) \leq\left(e_{2}-e_{1}\right)$ where

$$
f(\alpha)=\frac{\sqrt{3}}{\pi}\left[\ln (1-\alpha)+\frac{\alpha}{1-\alpha} \ln (\alpha)\right] .
$$

So, we can obtain the result which can be described in Figure 8 .

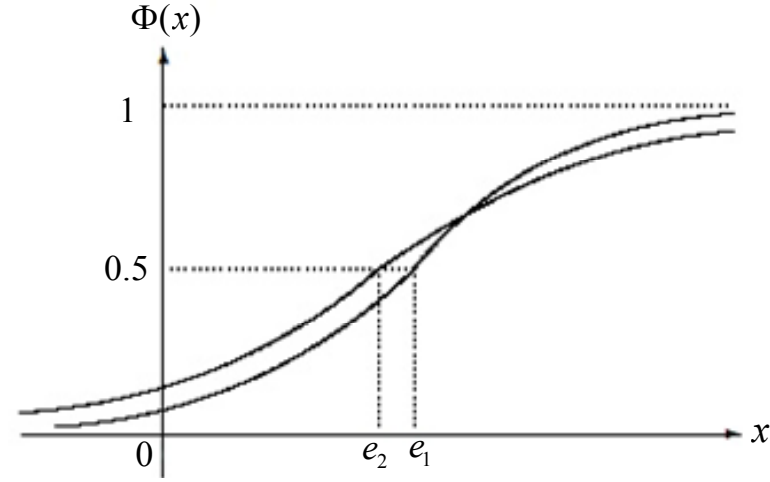

Figure 7. Second comparison of normal risks.

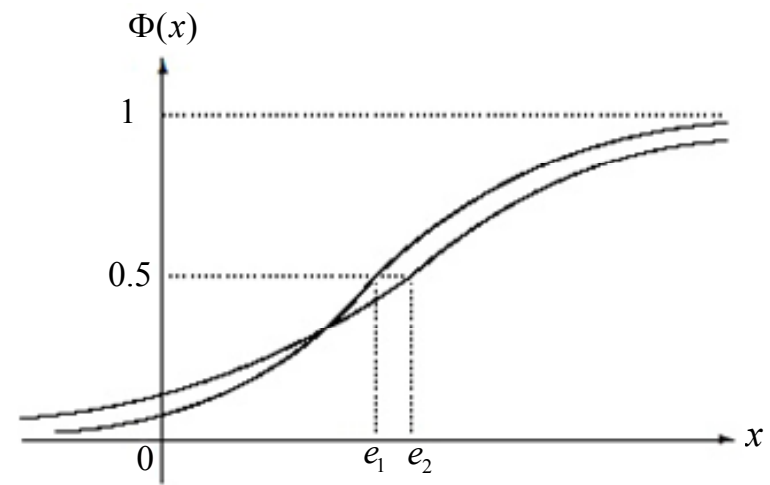

Figure 8. Third comparison of normal risks.

\section{CONCLUSION}

This paper has proposed a new approach to risk comparison by means of uncertain measures. We introduced the concepts of some uncertain measures and risk comparison rules within the framework of uncertain theory. Special attention is paid to building the bridges between uncertain measures and risk comparison rules. We described the three risk comparison rules by three kinds of uncertain measures: uncertain VaR, uncertain AVaR, and uncertain TVaR, respectively. Moreover, the results are applied in the risks with linear and normal uncertain distributions. This method will be a very helpful tool for studying risks whose characteristic is neither relative to randomness nor fuzziness. It should be emphasized that there are many ways to rank risks according to different uncertain measures. In risk analysis, choosing and finding a suitable risk measure to compare the risks will be constant exploration.

\section{ACKNOWLEDGMENTS}

This work is supported by the National Natural Science Foundation (No. 60874067), the Hubei Provincial Natural Science Foundation (No. 2010CDB02801), and 
the Scientific and Technological Innovation Team Project (No. T201110) of Hubei Provincial Department of Education, China.

\section{REFERENCES}

Artzner, P., Delbaen, F., Eber, J., and Heath, D. (1997), Thinking coherently, Risk, 10, 68-71.

Barrett, G. F. and Donald, S. G. (2003), Consistent tests for stochastic dominance, Econometrica, 71, 71-104.

Guldimann, T. M. (1995), Risk Metrics TM: Technical Document, JPMorgan, New York, NY.

Lee, E. S. and Li, R.-J. (1988), Comparison of fuzzy numbers based on the probability measure of fuzzy events, Computers and Mathematics with Applications, 15, 887-896.

Lee, L.-W. and Chen, S.-M. (2008), Fuzzy risk analysis based on fuzzy numbers with different shapes and different deviations, Expert Systems with Applications, 34, 2763-2771.

Liu, B. (2007), Uncertainty Theory (2nd ed.), Springer, Berlin, Germany.

Liu, B. (2009), Some research problems in uncertainty theory, Journal of Uncertain Systems, 3, 3-10.

Liu, B. (2010a), Uncertainty Theory: A Branch of Mathematics for Modeling Human Uncertainty, Springer, Berlin, Germany.

Liu, B. (2010b), Uncertain risk analysis and uncertain reliability analysis, Journal of Uncertain Systems, 4, 163-170.

Markowitz, H. (1952), Portfolio selection, The Journal of Finance, 7, 77-91.

Nojavan, M. and Ghazanfari, M. (2006), A fuzzy ranking method by desirability index, Journal of Intelligent and Fuzzy Systems, 17, 27-34.

Peng, J. (2008), Measuring fuzzy risk by credibilistic value at risk, Proceedings of the 3rd International Conference on Innovative Computing Information and Control, Dalian, China, 270.

Peng, J. (2009a), Value at risk and tail value at risk in uncertain environment, Proceedings of the 8th International Conference on Information and Management Sciences, Kunming, China, 787-793.

Peng, J. (2009b), Average value at risk in fuzzy risk analysis, Advances in Intelligent and Soft Computing, 62, 1303-1313.

Peng, J. and Li, S. (2010), Spectral measure of uncertain risk, Proceedings of the 1st International Conference on Uncertain Theory, Urumchi and Kashi, China, 1-7.

Peng, J. and Li, S. (2011), Distortion risk measures of uncertain systems, Proceedings of the 9th International Conference on Reliability, Maintainability and Safety, Guiyang, China, 460-467.

Peng, J., Jiang, Q., and Rao, C. (2007), Fuzzy dominance: a new approach for ranking fuzzy variables via credibility measure, International Journal of Uncertainty, Fuzziness and Knowledge-Based Systems, 15, 29-41.

Peng, J., Mok, H. M. K., and Tse, W.-M. (2005), Fuzzy dominance based on credibility distributions, Proceedings of the 2nd International Conference on Fuzzy Systems and Knowledge Discovery, Changsha, China, 295-303.

Shaked, M. and Shanthikumar, J. G. (1994), Stochastic Orders and Their Applications, Academic Press, Boston, MA.

Tran, L. and Duckstein, L. (2002), Comparison of fuzzy numbers using a fuzzy distance measure, Fuzzy Sets and Systems, 130, 331-341.

Zadeh, L. A. (1965), Fuzzy sets, Information and Control, 8, 338-353.

Zmeskal, Z. (2005), Value at risk methodology of international index portfolio under soft conditions (fuzzy-stochastic approach), International Review of Financial Analysis, 14, 263-275. 